\title{
1-Year Outcomes of 3-Month Dual Antiplatelet Therapy after Left Atrial Appendage Closure with the Watchman Occluder (the DUAL-WATCH study): a Post Hoc Analysis
}

\author{
Xiaoxia Liu ${ }^{1}$, Caroline Kleinecke ${ }^{2}$, Sonia Busch ${ }^{3}$, Wasim Allakkis ${ }^{4}$, Christian Mahnkopf ${ }^{3}$, \\ Johannes Brachmann ${ }^{3}$, Steffen Schnupp ${ }^{4}$, Steffen Gloekler ${ }^{5}$, Jiangtao $\mathrm{Yu}^{6}$, and Chang \\ Sheng $\mathrm{Ma}^{7}$ \\ ${ }^{1}$ Beijing Anzhen Hospital, Capital Medical University \\ ${ }^{2}$ Helmut-G.-Walther-Klinikum \\ ${ }^{3}$ Klinikum Coburg \\ ${ }^{4}$ Klinikum Coburg \\ ${ }^{5}$ University Hospital of Bern \\ ${ }^{6}$ Helmut-G.-Walther-Klinikum \\ 7 Beijing Anzhen Hospital, Capital Medical University
}

May 26, 2020

\begin{abstract}
Objective: Optimal antithrombotic therapy following left atrial appendage closure (LAAC) with the Watchman occluder (Boston Scientific) remains uncertain. This study aimed to investigate the efficacy and safety of a 3-month dual antiplatelet therapy (DAPT) after LAAC. Methods: This was a post hoc analysis of a prospective study of patients who underwent successful LAAC with Watchman devices at the REGIOMED Hospitals of Coburg and Lichtenfels (Germany). Those treated from 11/2016 to 05/2018 received DAPT for 3 months (DAPT group); those from 02/2012 to 04/2017 received 45-day anticoagulant+aspirin followed by 4.5-month DAPT (ACT group). The primary efficacy outcome and safety outcome were analyzed. The net clinical benefit and bleeding events 1 year after treatment, and their independent risk factors were also explored. Results: There were 220 and 304 patients in the DAPT and ACT groups. The primary efficacy outcome were 9.5\% vs. 6.3\% [hazard ratio (HR), 1.58; 95\% confidence interval (CI), 0.84-2.97; $\mathrm{P}=0.14$ ]; the primary safety outcome were $4.5 \%$ vs. $5.9 \%$ (HR, 0.80; 95\% CI, $0.38-1.69 ; \mathrm{P}=0.57)$; the net clinical benefit were $13.6 \%$ vs. $11.8 \%(\mathrm{HR}, 1.23 ; 95 \% \mathrm{CI}, 0.75-2.02 ; \mathrm{P}=0.39)$ over 1 year in DAPT and ACT groups, respectively. Age [?] 75 years (HR, 2.08; 95\%CI, 1.13-3.84; $\mathrm{P}=0.02)$ was identified as an independent predictor for the net clinical benefit. ACT (HR, 1.97; 95\% CI, 1.12-3.50; $\mathrm{P}=0.02)$ was independently associated with bleeding events after procedure. Conclusions: In patients who underwent LAAC using the Watchman occluder, 3-month DAPT is associated with fewer bleeding events compared with ACT regimen.
\end{abstract}

\section{List of abbreviations}

AF, Nonvalvular atrial fibrillation

ACT,Anticoagulation therapy

BARC, Bleeding Academic Research Consortium

BMI, Body mass index

CABG, Coronary artery bypass grafting 
CI, Confidence interval

CRNMB, Clinically relevant non-major bleeding

DAPT, Dual antiplatelet therapy

DRT, Device-related thrombus

HR, Hazard ratio

IS, Ischemic stroke

LAA, Left atrial appendage

LAAC, Left atrial appendage closure

$\mathrm{MB}$, major bleeding

NOAC, Non-vitamin K oral anticoagulant(s)

OAC, Oral anticoagulation

PCI, Percutaneous coronary intervention

SAPT, Single antiplatelet therapy

SE, Systemic embolism

TEE, Transesophageal echocardiography

TIA, Transient ischemic attack

VKA, Vitamin K antagonist(s)

\section{INTRODUCTION}

Atrial fibrillation (AF) is a common supraventricular tachyarrhythmia caused by uncoordinated atrial activation and associated with an irregularly irregular ventricular response ${ }^{1-4}$. The causes of AF include underlying structural heart disease, metabolic disorders, endocrine diseases, and certain medications ${ }^{1-4}$. The prevalence of $\mathrm{AF}$ is approximately $1 \%-2 \%$ in the general population in developed countries ${ }^{1-6}$. Patients with $\mathrm{AF}$ are often at a significantly increased risk of thromboembolism and, in particular, stroke ${ }^{1-4}$. Most patients with atrial fibrillation are at increased risk of stroke and should receive thromboembolic prophylaxis in order to lower that risk ${ }^{1-4}$. The risk of thromboembolism can be significantly reduced by appropriate use of antithrombotic therapy but at a somewhat increased risk of bleeding ${ }^{1-4}$.

Left atrial appendage closure (LAAC) has emerged as a valid non-pharmacological option for the prevention of stroke in patients with nonvalvular AF and contraindications to an indefinite oral anticoagulation (OAC) therapy ${ }^{2}$. Post-procedural antithrombotic therapy is usually administered to prevent device-related thrombi (DRT) and subsequent thromboembolic events until device endothelialization ${ }^{7}$. Nevertheless, each regimen carries a certain risk of bleeding complications. The optimal drug regimen and duration following LAAC with the Watchman occluder (Boston Scientific, Marlborough, MA, US) is still under investigation. As a consequence, various post-procedural anticoagulation strategies are adopted among centers ${ }^{7,8}$.

In the randomized PROTECT-AF and PREVAIL trials, the patients received a vitamin K antagonist (VKA) plus aspirin for 45 days, followed by dual antiplatelet therapy (DAPT) for 4.5 months in patients eligible for OAC after Watchman LACC ${ }^{9,10}$. Subsequent studies showed that DAPT for variable durations was an effective and safe alternative to warfarin after Watchman implantation in large real-world registries ${ }^{11,}$ 12. A single-center registry reported the feasibility of a shortened 6 -week DAPT ${ }^{13}$. In the multicenter EWOLUTION registry, patients on non-vitamin K oral anticoagulants (NOACs) had the lowest bleeding rate, without an increase in DRT or stroke rates ${ }^{14}$. Nevertheless, the most optimal strategy remains to be defined. 
This post hoc analysis of a dual-center prospective observational study examined the incidence rates of nonprocedure-related complications including death, thromboembolic events, and bleeding events in patients who received a 3-month DAPT regimen vs. 6-week anticoagulation plus aspirin followed by a 4.5-month DAPT (ACT regimen) over a 12-month follow-up period after successful LAAC. In addition, the associated risk factors for net clinical benefit and clinically relevant bleedings were examined.

\section{PATIENTS AND METHODS}

\section{Study design and patients}

This was a post hoc analysis of a prospective study of all consecutive patients who underwent LAAC with Watchman devices between 2012 and 2018 at the REGIOMED Hospitals of Coburg and Lichtenfels (Germany) and who were prospectively enrolled in an observational registry. The indications for LAAC were based on the current guidelines and recommendations ${ }^{15,16}$. The exclusion criteria were active infection, pregnancy, intracardiac thrombus, and reasons for OAC other than AF. All patients provided written informed consent. The study protocol conforms to the ethical guidelines of the Declaration of Helsinki as reflected in a priori approval by the institution's human research committee.

\section{Grouping}

For this post hoc analysis, the patients with successful LAAC performed between November 2016 and May 2018 and later treated with DAPT for 3 months were grouped as DAPT group. Those who underwent LAAC successfully between February 2012 and April 2017 and received anticoagulant plus aspirin for 45 days followed by DAPT for 4.5 months were grouped into ACT group.

LAAC, antithrombotic therapy, and TEE

The characteristics of the Watchman device (Boston Scientific, Marlborough, MA, US) and procedural aspects were previously described in detail ${ }^{17}$. The device was implanted via a transseptal approach using a delivery sheath. All procedures were guided by transesophageal echocardiography (TEE) and fluoroscopy.

At the Lichtenfels site, patient enrollment started in 2012. Antithrombotic therapy following LAAC was performed according to the PROTECT-AF trial ${ }^{10}$ and consisted of 45 days of aspirin plus OAC with a VKA or a NOAC (ACT group). Since the majority of the patients had contraindications to OAC, a fully dosed low molecular weight heparin was administered instead. In case of complete LAA closure without 45-day DRT or major ([?]5 $\mathrm{mm}$ ) peri-device leak, anticoagulation was stopped, and clopidogrel and aspirin were given until the final 6-month TEE control. In case of an unremarkable result, aspirin was continued indefinitely as a single antiplatelet therapy.

At the Coburg site, patient recruitment started in 2016, and the postprocedural antithrombotic regimen consisted of a short DAPT with aspirin and clopidogrel for 3 months only (DAPT group). In this group, a single follow-up TEE was performed after 3 months. In the case of DRT, the antithrombotic therapy was switched to OAC.

Follow-up

A systematic clinical follow-up after 12 months was carried out by outpatient visits, phone contact, or hospital stays. Demographic, clinical, and procedural characteristics, as well as adverse events and outcomes, were recorded according to the current recommendations of the European Associations of Percutaneous Coronary Interventions ${ }^{16}$, the Munich consensus document on definitions, endpoints and data collection requirements ${ }^{18}$, the Bleeding Academic Research Consortium (BARC) ${ }^{19}$, the clinically relevant non-major bleeding (CRNMB) definition defined by the Scientific and Standardization Committee of the International Society on Thrombosis and Haemostasis for the clinical trials, registries, and cohort studies of AF and venous thromboembolic disease ${ }^{20}$, the Valve Academic Research Consortium criteria ${ }^{21}$, and the 2017 Cardiovascular and Stroke Endpoint Definitions for Clinical Trials ${ }^{22}$. Device success was defined as the correct deployment and implantation of the Watchman device. 
The analysis of the demographic characteristics, procedural data, and clinical outcomes was performed for all patients in a standardized manner. Contemporary risk scores for thromboembolism $\left(\mathrm{CHA}_{2} \mathrm{DS}_{2}-\mathrm{VASc}\right)$ and bleeding (HAS-BLED) were calculated ${ }^{23,24}$.

\section{Outcomes}

The primary efficacy outcome was defined as a composite of the occurrence of ischemic stroke (IS), transient ischemic attack (TIA), systemic embolism (SE), and cardiovascular/unexplained death. The primary safety outcome referred to the occurrence of major bleeding $(\mathrm{MB})$ which was defined as BARC $>2$. The secondary safety outcome was the incidence of clinically relevant bleeding events, including MB and CRNMB. The combined hazard outcome (i.e., the net clinical benefit), was defined as the composite of the primary efficacy and primary safety outcomes. If a patient experienced more than one event(s) during follow-up, only the first event of a kind was considered. All the procedure-related events (bleeding due to vascular access and pericardial effusion, stroke/SE, or death [?]7 days within implantation), were not analyzed. Adverse events were adjudicated by a clinical event committee of two independent physicians, and, in case of disagreement, a third referee participated in the discussion.

Statistical analysis

Continuous variables were presented as means \pm standard deviations (SD), and categorical variables were presented as frequency and percentage. Clinical features between groups were compared using the Student's t-test for continuous measures and the chi-square test for categorical variables. The Kaplan-Meier method was used for graphical assessment of time-dependent events. The log-rank (Mantel-Cox) test was used to compare clinical event outcomes, and the Mantel-Haenszel method was applied to determine hazard ratios. Multivariable Cox regression model (enter method) was used to identify risk factors independently associated with the net clinical benefit and clinically relevant bleeding events, based on the results of the on-treatment analysis. These indicators with $\mathrm{P}<0.20$ in the univariable analyses were entered into a multivariable analysis. Multivariable Cox regression analyses were performed with SPSS 25.0 for Windows (IBM Corp, Armonk, NY, USA). All other statistical analyses were performed using GraphPad Prism 7 (GraphPad Inc., La Jolla, CA, USA). It was considered statistically significant when $\mathrm{P}<0.05$.

\section{RESULTS}

Characteristics of the patients

A total of 304 consecutive patients who underwent successful LAAC with the Watchman occluder between February 2012 and April 2017 at the Lichtenfels site were treated with the ACT regimen. A total of 220 patients at the Coburg site underwent Watchman implantation between November 2016 and May 2018 and received DAPT regimen. The baseline characteristics of the two groups are shown in Table 1 . The patients in the DAPT group were significantly older, less likely to have a history of liver dysfunction at baseline, and had lower left ventricular ejection fraction, more tendency to take drugs with a predisposition to bleeding and have a history of $\mathrm{MB}$ events. The mean $\mathrm{CHA}_{2} \mathrm{DS}_{2}$-VASc and HAS-BLED scores were $4.8 \pm 1.5$ and $3.3 \pm 0.7$ for the DAPT group, respectively, and $4.5 \pm 1.7$ and $3.4 \pm 1.0$ for the ACT group, respectively.

\section{Periprocedural aspects and TEE follow-up}

LAAC was successfully performed in all patients, and all $220(100.0 \%)$ patients in the DAPT group were discharged on DAPT. In the ACT group, 270 (88.8\%) patients were discharged on LMWH plus aspirin and 34 $(11.2 \%)$ patients on OAC plus aspirin. Procedure-related events are summarized in Table 2, and all patients were managed conservatively without sequelae. TEE was available for $190(86.4 \%)$ patients in the DAPT and $267(87.8 \%)$ in the ACT group, respectively (Table 3). The number of major ([?]5 $\mathrm{mm}$ ) peri-device leak was three (1.1\%) in the ACT group and two (1.1\%) in the DAPT group. Among those cases, one patient in the ACT group had TIA despite treatment with a NOAC. DRT was detected in 13/267 (4.9\%) patients in the ACT group and 5/190 (2.6\%) in the DAPT group. Among thirteen cases of DRT in the ACT group, ten received NOAC, and the others resumed aspirin plus warfarin. Of those, nine were resolved in repeat TEE exams. In the DAPT group, all five cases of DRT were treated with NOAC, and two were resolved according 
to TEE follow-up. In two cases, TEE control was refused. In both groups, none of the cases with DRT resulted in clinical adverse events. In the ACT group, DRT was associated with two ischaemic disabling strokes, despite the fact that one patient was still under OAC.

Clinical follow-up

The results of the 12-month follow-up are presented in Table 3 and Figure 1. In the DAPT group, OAC with VKA and NOAC was restarted in $14(6.4 \%)$ patients. Reasons were ischemic stroke $(\mathrm{n}=5)$, pulmonary embolism/deep vein thrombosis $(n=5)$, thrombus on the ventricular electrode of an indwelling implantable cardioverter-defibrillator $(\mathrm{n}=1)$, significant peri-device flow $(\mathrm{n}=2)$, and DRT $(\mathrm{n}=1)$. DAPT was prolonged to 12 months in nine $(4.1 \%)$ patients due to concomitant coronary heart disease. In the ACT group, 17 $(5.6 \%)$ patients resumed oral anticoagulation with VKA or NOACs. Reasons were ischemic stroke $(\mathrm{n}=8)$, pulmonary embolism/deep vein thrombosis $(n=6)$ and DRT $(n=3)$. Ten $(3.3 \%)$ were still on DAPT for coronary heart disease.

Primary efficacy outcome

The occurrence of primary efficacy outcome was similar in both groups. It was reached $9.5 \%(21 / 220)$ in DAPT group and 6.3\% (19/304) in ACT group [hazard ratio (HR), 1.58; $95 \%$ confidence interval (CI), 0.84-2.97; $\mathrm{P}=0.14]$. None of the components of the primary efficacy outcome was significantly different between the two groups. Thromboembolic events occurred in $3.2 \%(7 / 220)$ in DAPT and $3.6 \%(11 / 304)$ in ACT groups (HR, 0.94; 95\%CI, 0.37-2.41; $\mathrm{P}=0.90)$. In the DAPT group, seven patients experienced thromboembolic events (five ISs and two TIAs, incidence of $3.2 \%$ ), including one non-disabling ischemic stroke and one TIA documented during the first 3 months post LAAC, and one TIA, three disabling, and one non-disabling ischemic strokes that occurred under single aspirin therapy during follow-up. In the ACT group, 11 patients experienced thromboembolic events (eight ISs and three TIAs, incidence of 3.6\%). Among them, one non-disabling ischemic stroke occurred in the third month after the procedure, when the patient was on DAPT treatment. Three patients suffered from TIA and three patients from non-disabling and four disabling strokes under single aspirin therapy. The all-cause mortality was higher $(\mathrm{P}=0.02)$ in the DAPT group. A non-significant trend for a higher rate of cardiovascular and unexplained deaths $(\mathrm{P}=0.05)$ was observed in the DAPT group. See details in Table 3.

Primary safety outcome

The primary safety outcome occurred with a comparable frequency $(4.5 \%$ vs. $5.9 \%$; HR, 0.80; $95 \%$ CI, $0.38-$ 1.69; $\mathrm{P}=0.57$; Table 3). Among the 10 cases of MB events in the DAPT group, six cases of gastrointestinal bleeding and three of soft tissue bleeding occurred in the first 3 months after DAPT, of which one was fatal, and eight was severe. One soft tissue MB happened 248 days post-implant in a patient taking OAC due to deep vein thrombosis. In the ACT group, 18 cases of MB events, including major gastrointestinal bleeding $(n=4)$, fatal intracranial bleeding $(n=2)$, severe epistaxis $(n=1)$, and fatal spontaneous retroperitoneal hematoma $(\mathrm{n}=1)$ during the first 45 days after LAAC under ACT plus aspirin occurred. Eight patients suffered from major gastrointestinal bleeding, and another suffered from fatal intracranial bleeding under DAPT between 45 days and 6 months. One soft tissue MB was documented at 332 days post-implantation under prolonged DAPT due to recent PCI. All cases of intracranial bleeding and the spontaneous retroperitoneal hematoma were fatal.

Secondary safety outcome

The secondary safety outcome of any bleeding events was significantly lower $(7.7 \%$ vs. $14.8 \%$; HR, 0.53 ; 95\% CI, 0.32-0.88; $\mathrm{P}=0.02$; Table 3 ) in the DAPT group. Fewer patients under the DAPT treatment suffered from CRNMB (3.2\% vs. 8.9\%; HR, 0.37; 95\%CI, 0.19-0.73; $\mathrm{P}=0.01$; Table 3). Most of the bleeding events $(15 / 17,88.2 \%$ in the DAPT and $39 / 45,86.7 \%$ in the ACT groups) were recorded during the intensified antithrombotic period.

Net clinical benefit 
Considering all the above-mentioned components of the primary outcomes, the combined hazard outcome, i.e., the net clinical benefit was similar for both strategies $(13.6 \%$ vs. $11.8 \%$; HR, $1.23 ; 95 \%$ CI, 0.75-2.02; $\mathrm{P}=0.39$; Table 3 ).

Risk factors associated with the net clinical benefit and bleeding events

Detailed HRs for risk factors associated with the net clinical benefit were presented in Table 4. Multivariable analysis revealed that the DAPT was not the independent associated factor of the net clinical benefit, while the age [?]75 years ( $\mathrm{HR}, 2.08 ; 95 \% \mathrm{CI}, 1.13-3.84 ; \mathrm{P}=0.02)$ proven to be its independent risk factor.

As for the outcome of any bleeding events, the ACT regimen was considered as the independent risk factor $(\mathrm{HR}, 1.97 ; 95 \% \mathrm{CI}, 1.12-3.50 ; \mathrm{P}=0.02)$ of it from the multivariable analysis. No other independent risk or protective factors were found (Table 5 ).

\section{DISCUSSION}

Optimal antithrombotic therapy after LAAC with the Watchman occluder remains uncertain. This study aimed to investigate the efficacy and safety of a 3-month dual antiplatelet therapy (DAPT) after LAAC, as well as the risk factors that could affect the net clinical benefit and bleeding events. The results strongly suggest that in patients who underwent LAAC with the Watchman occluder, 3-month DAPT is associated with fewer bleeding events.

A suitable bridging antithrombotic protocol is essential to prevent thromboembolic complications during the endothelialization period of LAAC devices. The strategy of ACT adopted in the PROTECT-AF and PREVAIL trials ${ }^{9,10}$ has the most solid scientific evidence for effectiveness, but it limited to patients eligible for OAC. In contrast to clinical trials, the bridging protocols are very heterogeneous in everyday clinical practice ${ }^{8}$. The most commonly used strategy, although it is only based on observational evidence, is DAPT directly after LAAC for a limited period (6 weeks to 6 months), followed by antiplatelet monotherapy, and the regimen prescribed in the PROTECT-AF and PREVAIL trials was less adopted ${ }^{8}$.

In the present post hoc study of 524 patients who underwent successful LAAC with the Watchman occluder and with 12 months of follow-up, 3-month DAPT regimen was compared with the regimen of 45-day ACT plus aspirin followed by 4.5 -month DACT (totally 6 months) after LAAC. Despite the fact that the investigated population in the DAPT group was a high-risk patient group (mean age, 78.4+-7.2; $\mathrm{CHA}_{2} \mathrm{DS}_{2}$-VASc, $4.8+-$ 1.5; HAS-BLED, 3.3+-0.7), no significant difference was found in primary efficacy and safety outcomes and net clinical benefit events between the two type of antithrombotic regimens. The less aggressive bridging antithrombotic regimen with DAPT did not reduce the net clinical benefits after 12 months. Age [?]75 years was independently associated with an increased risk of decreased clinical net benefits while bridging antithrombotic therapy was not a significant risk factor after multivariable analysis. Those findings confirm the results of the EWOLUTION registry and another propensity-matched study based on several Watchman registries, which demonstrated similar efficacy and safety of both ACT and DAPT regimens ${ }^{11,12}$.

Before device endothelialization, DRT and ischemic stroke are dreaded complications. On the other hand, bleeding events, due to anticoagulation, play a substantial role after LAAC. Of note, the rate of the overall bleeding events was significantly higher in the ACT group than in the DAPT group, although no differences in MB during follow-up were observed. More patients under the ACT treatment suffered from CRNMB). We found that the ACT regimen was an independent risk factor for bleeding events. Despite differences between studies, whether about the characteristics or definitions of outcomes, prior history of bleeding as well as elderly people $>75$ years were identified as independent risk factors for bleeding events ${ }^{7}$. In the present study, there was only a trend toward an association between these two factors with bleeding events. This is likely due to the low numbers of patients. Consistent with the EWOLUTION trial, in both treatment arms, all fatal and most of the bleeding events occurred during the most aggressive phase of antithrombotic therapy $^{12,14}$.

Less aggressive antithrombotic postprocedural LAAC therapies would be appealing, potentially causing fewer bleeding complications, which, to this date, is the most common and challenging complication after LAAC ${ }^{25}$. 
The current Watchman instructions for use recommend an antithrombotic regimen tailored to each patient's individual stroke and bleeding risk, allowing either a 3-months DAPT or an OAC. Several studies investigated various antithrombotic schemes following LAAC. The real-world ASAP registry evaluated a 6-months DAPT in 150 patients undergoing LAAC with the Watchman and reported a low annual rate of ischemic stroke of $1.7 \%{ }^{26}$. Another all-comer single-center registry documented the same rate of ischemic stroke (1.7\%) with a short-term DAPT for 6 weeks following LAAC with Amplatzer and Watchman devices in 298 patients ${ }^{7}$. Furthermore, it revealed a low annual major bleeding rate of 3.9\%. Two registries with 110 and 76 patients investigated a SAPT after LAAC with Amplatzer devices ${ }^{27,} 28$. They reported a slightly higher stroke rate of $2.3 \%$ and $4.0 \%$, as well as a very low bleeding rate of $2.1 \%$ and $1.3 \%$. Lastly, the 3-months results of the EWOLUTION registry found no relevant differences in DAPT, VKA, NOAC, SAPT, or no therapy with regard to effectiveness and safety. Interestingly, NOAC therapy had the lowest event rate, numerically ${ }^{14}$.

The annual rates of ischemic stroke and MB in the present study were similar for both treatment arms while slightly higher than the aforementioned registries. This could partially be explained by higher risks for ischemic stroke and MBs, as depicted by the relatively high CHA2DS2-VASc (4.8+-1.5) and HAS-BLED scores (3.3+-0.7), and a shorter follow-up duration with most events occurring in the early phase after device implantation. In the ACT group, MBs, including three fatal events, were observed during early follow-up and occurred predominantly in patients with a high HAS-BLED score and prior bleeding events. This observation was also made in the PROTECT and PREVAIL studies, which used the ACT regimen and documented a relatively high estimated annual bleeding rate of $10.5 \%{ }^{9,10}$. In the long term, at five years, the overall annual risk of $\mathrm{MB}$ was reduced to $3.1 \%^{29}$.

The rate of major periprocedural complications (3.4\%) in the present study was in line with other studies (PREVAIL study: $4.2 \%^{9}$, CAP registry: $4.1 \%^{30}$, EWOLUTION registry: $2.7 \%^{31}$ ). TEE at follow-up revealed a low rate of major peri-device leaks for both groups, which is comparable to that reported in the EWOLUTION registry $(0.7 \%)^{31}$. In the ACT group, one case of TIA was documented in a patient with a major peri-device leak. An association of peri-device leaks and the occurrence of thromboembolic events has not been found yet ${ }^{32}$. The rate of DRT did not differ between the treatment arms. In contrast to this finding, the propensity-matched analysis of ACT and DAPT observed more DRT in the DAPT group ${ }^{11}$. All patients with major peri-device leaks and DRT were switched to OAC. In the ACT group, two ischemic events were associated with DRT during follow-up. Nevertheless, neither peri-device leak nor DRT was associated with clinical adverse events during follow-up in the DAPT group. This is likely a chance finding due to the small sample size of the study, low event rate, and different frequency for TEE follow-up. The impact of DRT on ischemic stroke is well documented in several studies ${ }^{32-34}$.

All-cause mortality in the DAPT group was considerable and higher than in the ACT group. This is probably due to the slightly higher age, rates of coronary heart disease and a significantly poorer left ventricular function in the DAPT group compared with the ACT group. Nonetheless, the DAPT subgroup of the EWOLUTION registry also reported a death rate of $10 \%$, which reflects the elderly, fragile, and multimorbid LAAC patient population in European countries. Individual patient's aspects like comorbidities, quality of life, and residual life expectancy should be taken into account when considering LAAC in octogenarians.

\section{LIMITATIONS}

The present study was a non-randomized, observational, post hoc, and retrospective dual-center study with a relatively small sample size. The slightly older age and higher rates of coronary heart disease and prior bleeding history were observed in the DAPT group, but DAPT regimen allowed similar net clinical benefit and fewer bleeding events in this study. TEE follow-up was not available for all patients, not analyzed in a central laboratory, and the timing was different between the two centers. This may have led to an underreporting of DRT and peri-device leaks for both treatment arms. Nevertheless, there were no differences in the rate of incomplete TEE follow-up between the ACT and DAPT group. Hence, potential adverse findings not discovered are supposed to be distributed similarly between the two groups.

\section{CONCLUSIONS}


Compared to ACT plus aspirin, 3-month DAPT following LAAC with the Watchman occluder appears to offer similar protection from thromboembolic events and a lower rate of bleeding events.

\section{References}

1.Fuster V, Ryden LE, Cannom DS, et al. ACC/AHA/ESC 2006 Guidelines for the Management of Patients with Atrial Fibrillation: a report of the American College of Cardiology/American Heart Association Task Force on Practice Guidelines and the European Society of Cardiology Committee for Practice Guidelines (Writing Committee to Revise the 2001 Guidelines for the Management of Patients With Atrial Fibrillation): developed in collaboration with the European Heart Rhythm Association and the Heart Rhythm Society. Circulation . 2006;114:e257-354.

2.Kirchhof P, Benussi S, Kotecha D, et al. 2016 ESC Guidelines for the management of atrial fibrillation developed in collaboration with EACTS.European journal of cardio-thoracic surgery : official journal of the European Association for Cardio-thoracic Surgery . 2016;50:e1-e88.

3.Healey JS, Parkash R, Pollak T, et al. Canadian Cardiovascular Society atrial fibrillation guidelines 2010: etiology and initial investigations. The Canadian journal of cardiology . 2011;27:31-37.

4.January CT, Wann LS, Alpert JS, et al. 2014 AHA/ACC/HRS guideline for the management of patients with atrial fibrillation: a report of the American College of Cardiology/American Heart Association Task Force on practice guidelines and the Heart Rhythm Society. Circulation . 2014;130:e199-267.

5.Jackson SL, Tong X, Yin X, et al. Emergency Department, Hospital Inpatient, and Mortality Burden of Atrial Fibrillation in the United States, 2006 to 2014. The American journal of cardiology . 2017;120:19661973.

6.DeWilde S, Carey IM, Emmas C, et al. Trends in the prevalence of diagnosed atrial fibrillation, its treatment with anticoagulation and predictors of such treatment in UK primary care. Heart . 2006;92:10641070 .

7.Chen S, Weise FK, Chun KRJ, et al. Antithrombotic strategies after interventional left atrial appendage closure: an update. Expert review of cardiovascular therapy . 2018;16:675-678.

8.Tilz RR, Potpara T, Chen J, et al. Left atrial appendage occluder implantation in Europe: indications and anticoagulation post-implantation. Results of the European Heart Rhythm Association Survey. Europace . 2017;19:1737-1742.

9.Holmes DR, Jr., Kar S, Price MJ, et al. Prospective randomized evaluation of the Watchman Left Atrial Appendage Closure device in patients with atrial fibrillation versus long-term warfarin therapy: the PREVAIL trial. J Am Coll Cardiol . 2014;64:1-12.

10.Holmes DR, Reddy VY, Turi ZG, et al. Percutaneous closure of the left atrial appendage versus warfarin therapy for prevention of stroke in patients with atrial fibrillation: a randomised non-inferiority trial.Lancet . 2009;374:534-542.

11.Sondergaard L, Wong YH, Reddy VY, et al. Propensity-Matched Comparison of Oral Anticoagulation Versus Antiplatelet Therapy After Left Atrial Appendage Closure With WATCHMAN. JACC Cardiovasc Interv . 2019;12:1055-1063.

12.Bergmann MW, Ince H, Kische S, et al. Real-world safety and efficacy of WATCHMAN LAA closure at one year in patients on dual antiplatelet therapy: results of the DAPT subgroup from the EWOLUTION all-comers study. EuroIntervention . 2018;13:2003-2011.

13.Weise FK, Bordignon S, Perrotta L, et al. Short-term dual antiplatelet therapy after interventional left atrial appendage closure with different devices. EuroIntervention . 2018;13:e2138-e2146.

14.Bergmann MW, Betts TR, Sievert H, et al. Safety and efficacy of early anticoagulation drug regimens after WATCHMAN left atrial appendage closure: three-month data from the EWOLUTION prospective, 
multicentre, monitored international WATCHMAN LAA closure registry.EuroIntervention . 2017;13:877884.

15.Kirchhof P, Benussi S, Kotecha D, et al. 2016 ESC Guidelines for the management of atrial fibrillation developed in collaboration with EACTS.Eur Heart J . 2016;37:2893-2962.

16.Meier B, Blaauw Y, Khattab AA, et al. EHRA/EAPCI expert consensus statement on catheter-based left atrial appendage occlusion.Europace . 2014;16:1397-1416.

17.Fountain RB, Holmes DR, Chandrasekaran K, et al. The PROTECT AF (WATCHMAN Left Atrial Appendage System for Embolic PROTECTion in Patients with Atrial Fibrillation) trial. Am Heart $J$. 2006;151:956-961.

18.Tzikas A, Holmes DR, Jr., Gafoor S, et al. Percutaneous left atrial appendage occlusion: the Munich consensus document on definitions, endpoints and data collection requirements for clinical studies.EuroIntervention . 2016;12:103-111.

19.Mehran R, Rao SV, Bhatt DL, et al. Standardized bleeding definitions for cardiovascular clinical trials: a consensus report from the Bleeding Academic Research Consortium. Circulation . 2011;123:2736-2747.

20.Kaatz S, Ahmad D, Spyropoulos AC, et al. Definition of clinically relevant non-major bleeding in studies of anticoagulants in atrial fibrillation and venous thromboembolic disease in non-surgical patients: communication from the SSC of the ISTH. J Thromb Haemost . 2015;13:2119-2126.

21.Kappetein AP, Head SJ, Genereux P, et al. Updated standardized endpoint definitions for transcatheter aortic valve implantation: the Valve Academic Research Consortium-2 consensus document.EuroIntervention . 2012;8:782-795.

22.Hicks KA, Mahaffey KW, Mehran R, et al. 2017 Cardiovascular and stroke endpoint definitions for clinical trials. J Am Coll Cardiol . 2018;71:1021-1034.

23.Friberg L, Rosenqvist M, Lip GY. Evaluation of risk stratification schemes for ischaemic stroke and bleeding in 182678 patients with atrial fibrillation: the Swedish Atrial Fibrillation cohort study.Eur Heart J . 2012;33:1500-1510.

24.Lip GY, Frison L, Halperin JL, et al. Comparative validation of a novel risk score for predicting bleeding risk in anticoagulated patients with atrial fibrillation: the HAS-BLED (Hypertension, Abnormal Renal/Liver Function, Stroke, Bleeding History or Predisposition, Labile INR, Elderly, Drugs/Alcohol Concomitantly) score. J Am Coll Cardiol . 2011;57:173-180.

25.Maarse M, Swaans MJ, Boersma LVA. Postprocedural Management: Anticoagulation and Beyond. Card Electrophysiol Clin . 2020;12:77-88.

26.Reddy VY, Mobius-Winkler S, Miller MA, et al. Left atrial appendage closure with the Watchman device in patients with a contraindication for oral anticoagulation: the ASAP study (ASA Plavix Feasibility Study With Watchman Left Atrial Appendage Closure Technology). J Am Coll Cardiol . 2013;61:2551-2556.

27.Korsholm K, Nielsen KM, Jensen JM, et al. Transcatheter left atrial appendage occlusion in patients with atrial fibrillation and a high bleeding risk using aspirin alone for post-implant antithrombotic therapy. EuroIntervention . 2017;12:2075-2082.

28.Jalal Z, Dinet ML, Combes N, et al. Percutaneous left atrial appendage closure followed by single antiplatelet therapy: Short- and mid-term outcomes. Arch Cardiovasc Dis . 2017;110:242-249.

29.Reddy VY, Doshi SK, Kar S, et al. 5-Year Outcomes After Left Atrial Appendage Closure: From the PREVAIL and PROTECT AF Trials. J Am Coll Cardiol . 2017;70:2964-2975.

30.Reddy VY, Holmes D, Doshi SK, et al. Safety of percutaneous left atrial appendage closure: results from the Watchman Left Atrial Appendage System for Embolic Protection in Patients with AF (PROTECT AF) 
clinical trial and the Continued Access Registry. Circulation . 2011;123:417-424.

31.Boersma LV, Schmidt B, Betts TR, et al. Implant success and safety of left atrial appendage closure with the WATCHMAN device: peri-procedural outcomes from the EWOLUTION registry. Eur Heart $J$. 2016;37:2465-2474.

32.Saw J, Tzikas A, Shakir S, et al. Incidence and Clinical Impact of Device-Associated Thrombus and PeriDevice Leak Following Left Atrial Appendage Closure With the Amplatzer Cardiac Plug. JACC Cardiovasc Interv . 2017;10:391-399.

33.Pracon R, Bangalore S, Dzielinska Z, et al. Device Thrombosis After Percutaneous Left Atrial Appendage Occlusion Is Related to Patient and Procedural Characteristics but Not to Duration of Postimplantation Dual Antiplatelet Therapy. Circ Cardiovasc Interv . 2018;11:e005997.

34.Fauchier L, Cinaud A, Brigadeau F, et al. Device-related thrombosis after percutaneous left atrial appendage ccclusion for atrial fibrillation. J Am Coll Cardiol . 2018;71:1528-1536.

Table 1. Baseline characteristics

\begin{tabular}{|c|c|c|}
\hline Variables & All $(\mathrm{n}=524)$ & $\operatorname{DAPT}(\mathrm{n}=220)$ \\
\hline Demographics and clinical features & Demographics and clinical features & Demographics and clini \\
\hline Age at LAAC (years), mean \pm SD & $76.6 \pm 7.6$ & $78.4 \pm 7.2$ \\
\hline Body mass index $\left(\mathrm{kg} / \mathrm{m}^{2}\right)$, mean $\pm \mathrm{SD}$ & $29.3 \pm 5.8$ & $29.3 \pm 6.3$ \\
\hline Female sex, n (\%) & $114(21.8)$ & $75(34.1)$ \\
\hline Coronary artery disease, $\mathrm{n}(\%)$ & $273(52.1)$ & $126(57.3)$ \\
\hline Prior PCI/CAGB, n (\%) & $220(42.0)$ & $95(43.2)$ \\
\hline Serum creatinine level $(\mathrm{mg} / \mathrm{dl})$, mean $\pm \mathrm{SD}$ & $1.4 \pm 0.7$ & $1.3 \pm 0.8$ \\
\hline Paroxysmal AF, n (\%) & $182(36.1)$ & $79(35.9)$ \\
\hline LV ejection fraction, $\%$ & $54.7 \pm 10.4$ & $52.4 \pm 12.2$ \\
\hline Stroke risk & Stroke risk & Stroke risk \\
\hline $\mathrm{CHA}_{2} \mathrm{DS}_{2}$-VASc score, mean $\pm \mathrm{SD}$ & $4.6 \pm 1.6$ & $4.8 \pm 1.5$ \\
\hline $\mathrm{CHADS}_{2}$ score, mean $\pm \mathrm{SD}$ & $3.0 \pm 1.1$ & $3.0 \pm 1.1$ \\
\hline Prior stroke/TIA/Systemic embolism, n (\%) & $123(23.5)$ & $44(20.0)$ \\
\hline Congestive heart failure, $\mathrm{n}(\%)$ & $167(31.9)$ & $78(35.5)$ \\
\hline Arterial hypertension, $\mathrm{n}(\%)$ & $485(92.6)$ & $207(94.1)$ \\
\hline Age [?]75 years, $\mathrm{n}(\%)$ & $347(66.2)$ & $166(75.5)$ \\
\hline Vascular disease, n (\%) & $324(61.8)$ & $137(62.3)$ \\
\hline Diabetes mellitus, n (\%) & $173(33.0)$ & $75(34.1)$ \\
\hline Bleeding risk & Bleeding risk & Bleeding risk \\
\hline HAS-BLED score, mean \pm SD & $3.3 \pm 0.9$ & $3.3 \pm 0.7$ \\
\hline Age [?]65 years, n $(\%)$ & $492(93.9)$ & $216(98.2)$ \\
\hline History of hemorrhagic/bleeding tendencies, n (\%) & $435(83.8)$ & $184(83.6)$ \\
\hline Prior bleeding ([?]major), n (\%) & $309(59.0)$ & $142(64.6)$ \\
\hline Intracranial, n (\%) & $41(7.8)$ & $13(5.9)$ \\
\hline Gastrointestinal, n (\%) & $149(28.4)$ & $62(28.2)$ \\
\hline Other site, $\mathrm{n}(\%)$ & $119(22.7)$ & $67(30.5)$ \\
\hline Arterial hypertension [?]160mmHg, n (\%) & $41(7.8)$ & $18(8.2)$ \\
\hline Renal dysfunction, n (\%) & $40(7.6)$ & $11(5.0)$ \\
\hline Liver dysfunction, n (\%) & $47(9.0)$ & $9(4.1)$ \\
\hline Labile INR, n (\%) & $26(5.0)$ & $13(5.9)$ \\
\hline Drugs with predisposition to bleeding, n (\%) & $489(93.3)$ & $214(97.3)$ \\
\hline Alcohol intake $>8 \mathrm{U} /$ week, n (\%) & $49(9.4)$ & $17(7.7)$ \\
\hline
\end{tabular}


LAAC: left atrial appendage closure; DAPT: dual antiplatelet therapy; ACT: anticoagulant therapy; PCI: percutaneous coronary intervention; CABG: coronary artery bypass grafting; AF: atrial fibrillation; LV: left ventricle; TIA: transient ischemic attack; INR: international normalized ratio; SD: standard deviation.

Table 2. Peri-procedural outcomes (0-7 days)

\begin{tabular}{lll}
\hline Variables & All $(\mathrm{n}=524)$ & DAPT $(\mathrm{n}=220$ \\
\hline Major peri-procedural complications & Major peri-procedural complications & Major peri- \\
Death, immediate peri-procedural (any), n (\%) & $0(0.0)$ & $0(0.0)$ \\
Need for cardio-pulmonary resuscitation, n (\%) & $3(0.6)$ & $3(1.4)$ \\
Pericardial effusion requiring intervention, n (\%) & $5(1.0)$ & $2(0.9)$ \\
Stroke (any), n (\%) & $2(0.4)$ & $0(0.0)$ \\
Major bleeding ([?]BARC 3a), n (\%) & $5(1.0)$ & $2(0.9)$ \\
Need for bailout surgery, n (\%) & $0(0.0)$ & $0(0.0)$ \\
Device embolization, n (\%) & $2(0.4)$ & $1(0.5)$ \\
Acute echocardiographic outcomes & Acute echocardiographic outcomes & Acute echoc \\
Residual flow, n (\%) & $8(1.5)$ & $3(1.4)$ \\
Significant flow, n (\%) & $0(0.0)$ & $0(0.0)$ \\
DRT, n (\%) & $1(0.2)$ & $0(0.0)$ \\
Anti-thrombotic medical therapy post LAAC & Anti-thrombotic medical therapy post LAAC & Anti-thromb \\
Any oral anticoagulation, n (\%) & $270(51.5)$ & $0(0.0)$ \\
Vitamin K antagonists, n (\%) & $259(49.4)$ & $0(0.0)$ \\
Non-vitamin K antagonists, n (\%) & $11(2.1)$ & $0(0.0)$ \\
Low molecular weight heparin, n (\%) & $34(6.5)$ & $0(0.0)$ \\
Aspirin, n (\%) & $524(100.0)$ & $220(100.0)$ \\
Platelet inhibitors other than aspirin, n (\%) & $220(42.0)$ & $220(100.0)$ \\
\hline
\end{tabular}

DAPT: dual antiplatelet therapy; ACT: anticoagulant therapy; BARC: Bleeding Academic Research Consortium; DRT: device-related thrombus.

Table 3. 12-month ( $365 \pm 15$ days) follow-up outcomes

\begin{tabular}{|c|c|c|}
\hline Variables & All $(\mathrm{n}=524)$ & DAP \\
\hline Follow-up available, n (\%) & $524(100.0)$ & 220 \\
\hline TEE & TEE & TEF \\
\hline No. of TEE, n (\%) & $457(87.2)$ & 190 \\
\hline Residual flow, n (\%) & $33(6.3)$ & $10 /$ \\
\hline Significant flow, n (\%) & $5(1.0)$ & $2 /$ \\
\hline DRT, n $(\%)$ & $18(3.4)$ & $5 /$ \\
\hline Anti-thrombotic therapy at the time of follow-up & Anti-thrombotic therapy at the time of follow-up & An \\
\hline Any anticoagulation, n (\%) & $31(5.9)$ & 14 \\
\hline Vitamin $\mathrm{K}$ antagonists, n (\%) & $4(0.7)$ & $2(c$ \\
\hline Non-vitamin $\mathrm{K}$ antagonists, n (\%) & $27(5.2)$ & 12( \\
\hline Single anti-platelet, n (\%) & $464(88.5)$ & 188 \\
\hline Dual anti-platelet, n (\%) & $19(3.6)$ & 9( \\
\hline Anticoagulant plus anti-platelet, n (\%) & $0(0.0)$ & 0( \\
\hline No anti-thrombus therapy, n (\%) & $10(1.9)$ & 9( \\
\hline \multirow[t]{3}{*}{ Clinical events outcome } & Clinical events outcome & Cli \\
\hline & All & DAF \\
\hline & $\mathrm{n}=524$ & \\
\hline
\end{tabular}




\begin{tabular}{|c|c|c|}
\hline Variables & All $(\mathrm{n}=524)$ & DAP \\
\hline Primary efficacy outcome & $40(7.6)$ & $21(9$ \\
\hline Primary safety outcome & $28(5.3)$ & 10( \\
\hline Net clinical benefit, n (\%) & $66(12.6)$ & $30(1$ \\
\hline Death & Death & Dea \\
\hline All-cause death, n (\%) & $45(8.6)$ & $26(1$ \\
\hline Cardiovascular/Unexplained death, n (\%) & $23(4.4)$ & $14(6$ \\
\hline Non-cardiovascular death, n (\%) & $22(4.2)$ & 12( \\
\hline Ischemic stroke/tia/se & Ischemic stroke/tia/se & Isc \\
\hline Ischemic stroke/TIA/SE, n (\%) & $18(3.4)$ & 7( \\
\hline Ischemic stroke, n (\%) & $13(2.5)$ & 5( \\
\hline Disabling stroke, n (\%) & $7(1.3)$ & 3( \\
\hline Non-disabling stroke, n (\%) & $6(1.1)$ & 2( \\
\hline TIA, n $(\%)$ & $5(1.0)$ & 2( \\
\hline $\mathrm{SE}, \mathrm{n}(\%)$ & $0(0.0)$ & 0( \\
\hline Bleedings & Bleedings & Ble \\
\hline Any bleeding & $62(11.8)$ & 17 \\
\hline Major bleeding, n (\%) & $28(5.3)$ & 10 \\
\hline Intracranial, n (\%) & $3(0.5)$ & $0(0$. \\
\hline Gastrointestinal, n (\%) & $19(3.6)$ & $7(3$. \\
\hline Other sites, n (\%) & $6(1.1)$ & $3(1$. \\
\hline CRNMB, n (\%) & $34(6.5)$ & $7(3$. \\
\hline
\end{tabular}

DAPT: dual antiplatelet therapy; ACT: anticoagulant therapy; HR (95\%CI): hazard ratio (95\% confidence interval); TEE: Transesophageal echocardiography; DRT: device-related thrombus; TIA: transient ischemic attack; SE: systemic embolism; CRNMB: clinically relevant non-major bleeding.

\# HR here refers to the hazard ratio of the outcome index in the DAPT group to the ACT group.

* Mantel-Haenszel method to get the P-value for HR.

Table 4. Individual predictors associated with the net clinical benefit in the on-treatment analysis

\begin{tabular}{lllll}
\hline Variables & Univariable & Univariable & Multivariable & Multivariable \\
\hline & HR (95\%CI) & P-value & HR (95\%CI) & P-value \\
DAPT regimen & $1.21(0.74-1.96)$ & 0.45 & $1.05(0.64-1.71)$ & 0.86 \\
Age at time of implantation (years) & $1.05(1.02-1.09)$ & 0.01 & & \\
Age [?]75 years & $2.16(1.18-3.97)$ & 0.01 & $2.08(1.13-3.84)$ & 0.02 \\
Female gender & $0.98(0.60-1.62)$ & 0.94 & & \\
Body mass index & $0.98(0.94-1.03)$ & 0.47 & & \\
Persistent AF & $1.07(0.64-1.78)$ & 0.80 & & \\
Congestive heart failure & $1.10(0.66-1.84)$ & 0.71 & & \\
Hypertension & $1.00(0.40-2.48)$ & $>0.99$ & & \\
Diabetes mellitus & $1.55(0.95-2.53)$ & 0.08 & $1.55(0.95-2.53)$ & 0.08 \\
Prior stroke/TIA/SE & $1.15(0.66-2.00)$ & 0.62 & & \\
Vascular disease & $1.15(0.70-1.91)$ & 0.58 & & \\
Renal dysfunction & $0.38(0.09-1.55)$ & 0.18 & $0.39(0.09-1.58)$ & 0.19 \\
Liver dysfunction & $1.03(0.45-2.39)$ & 0.94 & & \\
History of bleeding & $0.95(0.50-1.82)$ & 0.88 & & \\
Labile INR & $0.92(0.29-2.92)$ & 0.89 & & \\
Alcohol intake $>8$ U/week & $1.20(0.55-2.62)$ & 0.65 & & \\
\hline
\end{tabular}


HR (95\%CI): hazard ratio (95\% confidence interval); DAPT: dual antiplatelet therapy; AF: atrial fibrillation; TIA: transient ischemic attack; SE: systemic embolism; INR: international normalized ratio.

Table 5. Individual predictors associated with bleeding events after LAAC in the on-treatment analysis

\begin{tabular}{lllll}
\hline Variables & Univariable & Univariable & Multivariable & Multivariable \\
\hline & HR $(95 \% \mathrm{CI})$ & P-value & HR $(95 \% \mathrm{CI})$ & P-value \\
ACT regimen & $1.88(1.07-3.28)$ & 0.03 & $1.97(1.12-3.50)$ & 0.02 \\
Age [?]65 years & $0.99(0.36-2.72)$ & 0.98 & & \\
Age [?]75 years & $1.52(0.86-2.69)$ & 0.15 & $1.76(0.98-3.15)$ & 0.06 \\
Female gender & $0.89(0.53-1.49)$ & 0.65 & & \\
Body mass index & $0.99(0.95-1.04)$ & 0.78 & & \\
Congestive heart failure & $1.08(0.63-1.84)$ & 0.78 & & \\
Hypertension & $0.78(0.33-1.80)$ & 0.56 & & \\
Diabetes mellitus & $1.31(0.79-2.19)$ & 0.29 & & \\
Prior stroke/TIA/SE & $1.25(0.71-2.18)$ & 0.44 & & \\
Vascular disease & $0.98(0.59-1.63)$ & 0.95 & & \\
Renal dysfunction & $1.10(0.44-2.74)$ & 0.84 & & \\
Liver dysfunction & $1.76(0.87-3.57)$ & 0.12 & $1.25(0.51-3.08)$ & 0.63 \\
History of bleeding & $2.23(0.89-5.56)$ & 0.09 & $2.08(0.83-5.20)$ & 0.12 \\
Labile INR & $1.37(0.50-3.76)$ & 0.55 & & \\
Alcohol intake >8 U/week & $1.74(0.86-3.52)$ & 0.13 & $1.66(0.68-4.05)$ & 0.27 \\
\hline
\end{tabular}

HR (95\% CI): hazard ratio (95\% confidence interval); ACT: anticoagulant therapy; TIA: transient ischemic attack; SE: systemic embolism; INR: international normalized ratio.

\section{Figure Legend}

Figure 1. Kaplan-Meier curves of the clinical outcomes of (A) the net clinical benefit, (B) the primary efficacy outcome, (C) the primary safety outcome, and (D) the secondary safety outcome of bleeding events at 12 months. DAPT: dual antiplatelet therapy; ACT: anticoagulant therapy.
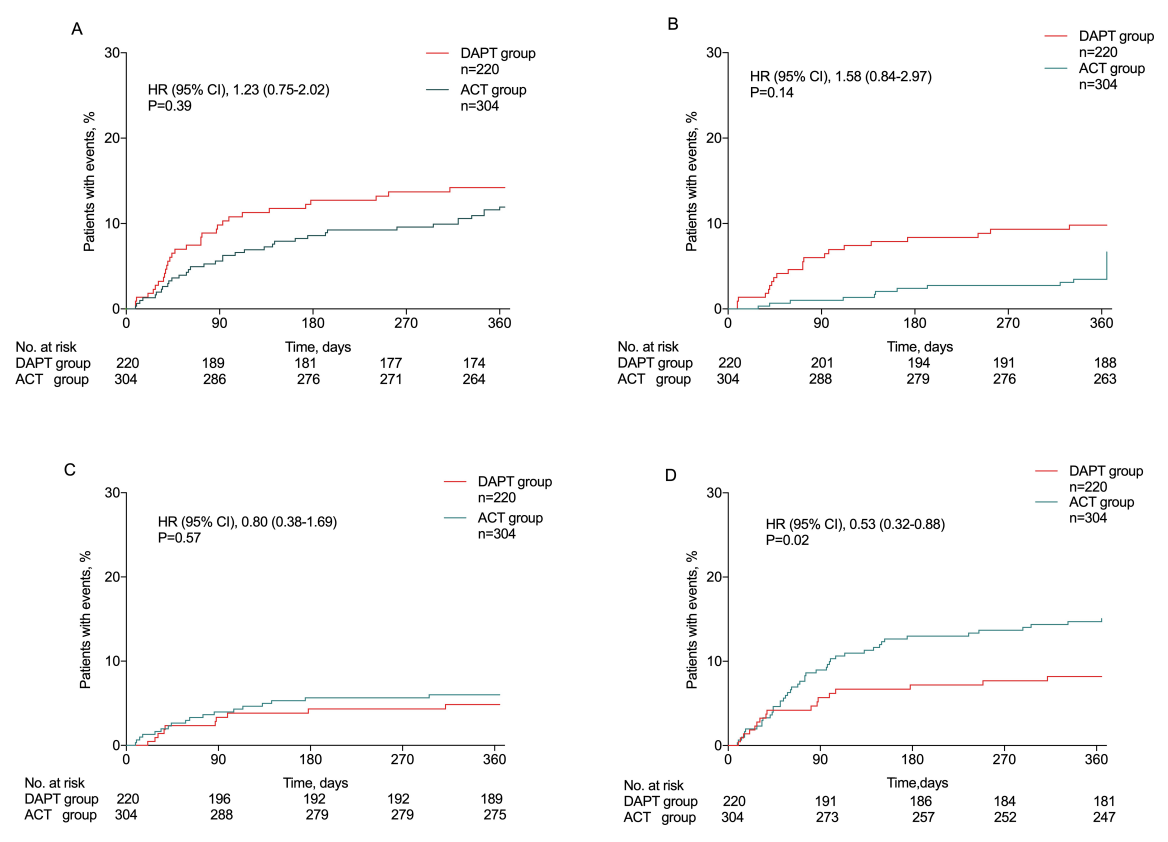